\title{
Paradoxical embolism associated with oral contraceptives: an underdiagnosed lesion?
}

\author{
J. DE SWIET \\ M.D., F.R.C.P. \\ East Glamorgan General Hospital, South Wales
}

\begin{abstract}
Summary
A case of cerebral embolism is described in which paradoxical features have been proved clinically, and which may explain some hitherto unaccountable reported cases, and suggests the value of prospective studies. A successful outcome of such a proved case, associated with oral contraceptives, does not appear to have been reported previously.
\end{abstract}

\section{Case report}

A 23-year-old right-handed married woman, who had been taking Minovlar continuously for 3 years, was admitted to hospital $24 \mathrm{hr}$ after the sudden onset, while walking, of a complete right-sided hemiplegia and total motor aphasia. She was a nonsmoker, and there was no previous history of any illness, nor any family history of cardiovascular disease. Her blood pressure was $120 / 80 \mathrm{mmHg}$; there was a regular tachycardia of 150 beats $/ \mathrm{min}$, a loud pulmonary component of $\mathbf{S 2}$, and a grade $3 / 6$ ejection systolic murmur at the pulmonary area. There was no clinical evidence of deep vein thrombosis in either leg. An ECG on admission confirmed a sinus tachycardia of 150 beats/min with a deep $\mathrm{S}$ wave in lead 1, a small Q3, and 'staircase' S-T segments in leads 2, 3, VF and V2-V6 inclusive. The transition zone was at V6, showing marked clockwise rotation. These features, indicating acute cor pulmonale from massive pulmonary embolism, had completely disappeared when the ECG was repeated 2 days later. The following investigations were normal: haemoglobin, WBC, ESR, cerebrospinal fluid, SMA12 biochemical profile, serum lipoproteins, urinalysis and chest X-ray.

Bilateral leg venography one week after admission showed a normal left leg, but the right showed nonfilling of the deep veins of the calf. At right-heart catherization an atrial communication was crossed, which was proved to be a patent foramen ovale by pulmonary artery pressures and dilution indicator curves.

A clinical diagnosis of paradoxical embolism was made on the grounds of the combined evidence of deep vein thrombosis, ECG changes of acute cor pulmonale (entirely different from those known to accompany primary cerebrovascular lesions) and catheter studies typical of patent foramen ovale. The clinical circumstances of the cerebrovascular lesion were also typical of embolism rather than thrombosis.

In view of the gross neurological deficit it was felt that anti-coagulation was contra-indicated, and the right femoral vein was ligated prophylactically. Recovery of speech and of function of the right arm and leg was eventually complete.

\section{Discussion}

Cerebral, coronary or other arterial occlusions in women taking oral contraceptives have usually been attributed to local thrombosis, although some authors have postulated embolism as the aetiology, without proving the source during life. The younger the patient the more likely is a sudden arterial occlusion to be embolic rather than thrombotic, the usual source being in the left heart from lesions easily excluded clinically and by echocardiography, leaving 'cryptogenic' cases in women taking oral contraceptives as described by Enzell and Lindemalm (1973), and by Ask-Upmark and Bickerstaff (1976). In an important and extensive retrospective analysis of strokes in women taking oral contraceptives (Collaborative Group Study, 1973) the protocol excluded from a diagnosis of embolism cases categorized as uncertain infarction, thrombotic infarction, stroke, or transient cerebral ischaemia: a diagnostic possibility of paradoxical embolism was not considered, although it could have explained some of the above categories (as defined in the protocol).

Meister et al. (1972) discussed the diagnosis of paradoxical embolism during life, describing 5 cases, one of them taking oral contraceptives and known to have a large atrial septal defect; but confirmation of thrombophlebitis in both popliteal veins was only made at post-mortem. 
The reported increased incidence of cerebral and coronary artery thrombosis in women taking oral contraceptives may need revision in the light of the present and other cases, as they may merely reflect a complication of primarily venous thrombosis in patients having a patent foramen ovale. Prospective studies to reveal paradoxical embolism in such cases may be rewarding, especially as Wood (1968) stated that valvular patency of the foramen ovale is present in about a third of all individuals.

\section{Acknowledgment}

I am grateful to Dr M. Ruttley, Department of Radiology,
University Hospital of Wales, for his report on the catheterization studies.

\section{References}

Ask-Upmark, E. \& Bickerstaff, E.R. (1976) Vertebral artery occlusion and oral contraceptives. British Medical Journal, 1, 487.

Collaborative Group for the Study of Stroke in Young WOMEn (1973) New England Journal of Medicine, 288, 871.

Enzell, K. \& Lindemalm, G. (1973) Cryptogenic cerebral embolism in women taking oral contraceptives. British Medical Journal, 4, 507.

Meister, S.G., Grossman, W., Dexter, L. \& Dalen, J.E. (1972) Paradoxical embolism. American Journal of Medicine, 53, 292.

Wood, P. (1968) Diseases of the Heart and Circulation, 3rd edn, p. 958. Eyre \& Spottiswoode, London. 